\title{
A influência da aglomeração e da concentração da indústria sobre a produtividade total dos fatores das empresas industriais brasileiras
}

Palavras-chave

Produtividade,

Competências para

Inovar, Aglomeração,

Concentração de mercado

Classificação JEL

D04, O31, O33, R11.

\section{Keywords}

Productivity,

Innovative Capabilities, Agglomeration, Market Concentration.

\section{JEL Classification}

D04, O31, O33, R11.

Programa de Pós-Graduação em Economia - PPGE - Universidade Federal de Santa Catarina (UFSC), Florianópolis/SC.

**Programa de Pós-Graduação em Desenvolvimento Econômico PPGDE - Universidade Federal do Paraná (UFPR), Curitiba/PR.

\section{Resumo}

Este artigo identifica a relação entre a Produtividade Total dos Fatores (PTF) da empresa, suas competências, a aglomeração e a concentração setoriais, contribuindo com a discussão sobre os Sistemas Setoriais de Inovação. Dada a relação próxima entre os setores e a localização espacial, bem como a hipótese Schumpeteriana de maior capacidade de inovação das empresas maiores (mercado concentrado), um modelo de regressão multinível considera a relação entre a PTF da empresa por suas competências no primeiro nível e em função das variáveis setoriais no segundo nível. Os efeitos estimados mostram que a aglomeração e a concentração juntamente com as competências internas da empresa explicam as diferenças de produtividade entre as empresas. Os resultados também mostram que alguns setores são mais sensíveis a aglomeração e a concentração setoriais sobre a produtividade e a dinâmica de inovação da empresa, todavia, os resultados não se alinham com a taxonomia de Pavitt (1984) e Dosi, Pavitt e Soete (1990).

\author{
Ronivaldo Steingraber \\ Flávio de Oliveira Gonçalves ${ }^{* *}$
}

\section{Abstract}

This article investigates the relationship between Total Factor Productivity (TFP) of a company, its capabilities, the agglomeration and sectoral concentration, contributing to the discussion of Sectoral Innovation Systems. Given the close relationship between the sectors and their spatial location, as well as the Schumpeterian hypothesis of greater innovativeness of large companies (market focused), a multilevel regression model considers the relationship of the TFP of a company according to its capabilities on the first level and the sectorial variables on the second level. The estimated effects show that the agglomeration and concentration together with the internal capabilities of a company explain productivity differences between companies. The results also show that some sectors are more sensitive to sectorial agglomeration and concentration on the company's productivity and innovation dynamics. However, the results do not align with the taxonomy of Pavitt (1984) and Dosi, Pavitt and Soete (1990). 


\section{1_Introdução}

O processo de inovação ganha forma e é visualizado na empresa, mas sua dinâmica não se restringe a este ambiente, pois envolve diversos agentes econômicos e institucionais nas suas diferentes etapas. A empresa inovadora apresenta ganhos de produtividade relacionados ao processo de inovação. No mesmo passo, percebe-se a formação de capital social entre a empresa inovadora e os atores sociais e econômicos necessários para alavancar o sucesso da inovação.

A relação entre a empresa e a estrutura envolvida com a inovação muda de setor para setor. Percebe-se que muitas competências para inovar são diferentes estrategicamente conforme o perfil tecnológico da indústria em questão. Neste sentido, o conceito de sistemas de inovação mostra que a estrutura de inovação na economia pode apresentar diferentes configurações.

Este artigo busca identificar como se dá a relação entre a empresa e o setor, contribuindo com a discussão sobre os Sistemas Setoriais de Inovação da literatura Schumpeteriana. Todavia, quando se discute a concentração setorial de empresas, no sentido de que setores com maiores empresas inovam mais e apresentam maior poder de mercado, verifica-se que a relação entre a empresa e importantes instituições do processo de inovação, como universidade e centros de pesquisa, além do tamanho da empresa, depende da concentração espacial, o que mostra que o capital social se estabelece territorialmente no processo de inovação.

Desta forma, uma discussão importante para os Sistemas Setoriais de Inovação é a relação entre a aglomeração espacial e o impacto sobre a inovação e a produtividade da empresa. A contribuição deste artigo está na análise empírica desta relação, separando o impacto microeconômico (competências internas para inovar) e o impacto setorial (aglomeração espacial e concentração) sobre a produtividade total dos fatores das empresas industriais brasileiras no ano de $2005^{1}$.
O modelo não considera a inovação de forma direta, mas sim, a produtividade da empresa. A relação entre as duas variáveis é direta e interdependente. A escolha da produtividade total dos fatores é justificada em função da maior abrangência desta variável em comparação com a inovação. Todas as empresas industriais brasileiras podem ser consideradas no modelo, o que nos garante que o total da população de empresas industriais brasileiras está analisado no modelo. A inovação é considerada nas competências internas da empresa. Como a produtividade não depende exclusivamente da inovação, outras variáveis relacionadas ao comércio exterior, escala, investimentos e perfil geral do capital humano são introduzidas em primeiro nível do modelo. $O$ impacto setorial da aglomeração e da concentração de mercado é considerado no segundo nível do modelo. A interpretação do resultado estimado está restrita à produtividade total dos fatores (PTF) e sua relação com a série utilizada de variáveis. Os resultados estimados corroboram a proximidade entre os ganhos de produtividade e o sucesso da inovação.

Neste sentido, este artigo também contribui com a discussão dos modelos Neoclássicos de crescimento endógeno, na medida em que se introduz a PTF (estatisticamente significativa) como medida generalista do desempenho tecnológico da empresa, explicada estatisticamente por uma série de variáveis da empresa e do setor, algumas relacionadas com a capacidade de inovação, mas também com a estrutura de mercado e demais competências internas da empresa.

Econometricamente, o exercício da estimativa foi realizado por meio de um modelo de regressão hierárquico (ou multinível). Mostramos como as competências para inovação explicam a produtividade total dos fatores nas empresas industriais brasileiras no ano de 2005. Este é o primeiro exercício que corresponde ao primeiro nível do modelo 
multinível. O segundo nível estimado capta a relação entre as variáveis setoriais sobre a empresa (suas competências para inovação e a produtividade). Para explicar o efeito da aglomeração espacial da indústria sobre as empresas introduz-se uma variável de aglomeração por estados na indústria brasileira. Para explicar o efeito da concentração setorial sobre as empresas, utilizamos o HHI (Hirschman Herfindahl Index).

Os resultados encontrados afirmam que a empresa (suas competências para inovar) e o setor impactam de forma diferente a produtividade da empresa. Os resultados também mostram que alguns setores apresentam a PTF das empresas mais sensíveis a aglomeração e concentração setorial, identificam-se diferenças setoriais para a capacidade de inovação na indústria brasileira, fato que contribui com a discussão de Política Industrial e Tecnológica na indústria brasileira.

Este artigo é composto de cinco seções, contando com a introdução e a conclusão. A seção dois analisa a teoria relacionada aos efeitos da concentração espacial e setorial sobre a produtividade e a capacidade de inovação das empresas. A seção três analisa o modelo econométrico desenvolvido. A seção quatro apresenta e discute os resultados estimados.

\section{2_Concentração e Aglomeração na Indústria}

A literatura Schumpeteriana de Sistemas de Inovação advoga que o processo de inovação depende da interação das competências internas para inovar da empresa com o ambiente. Carlsson et alli (2002) apresentam quatro sistemas nessa linha de pesquisa: regional, nacional, setorial e tecnológico.

O conceito de sistema significa relacionamento e articulação entre os componentes do todo, bem como os atributos e competências de relacionamento presentes entre os participantes.
Considerando-se o Sistema Setorial de Inovação (SSI), apresentado nos trabalhos de Breschi e Malerba (1997) e Malerba (2002), pode-se defini-lo como a articulação entre inovação e a estrutura de mercado. Nesse sentido, a estrutura de mercado do setor considerada vai além da visão de estrutura de mercado, com os componentes de integração vertical ou diversificação. O olhar do SSI é mais focado nas relações entre os agentes e as características do setor, como o perfil do produto, agentes, processos de conhecimento e aprendizado, tecnologias básicas, insumos, demanda, links relacionados e complementares, mecanismos de interação entre empresas e outras empresas de fora, processo de competição e seleção e instituições presentes na esfera setorial da economia.

O foco do SSI está mais na mudança estrutural do setor, dado o avanço do progresso tecnológico e das inovações. Apesar do foco na dinâmica, algumas regularidades podem ser observadas no sistema ao longo do tempo. A primeira regularidade é a inter-relação com a dimensão regional, o que aproxima mais o SSI do estudo do Sistema Regional de Inovação (SRI) do que do Sistema Nacional de Inovação (SNI), conforme aponta Malerba (2002) a ênfase regional (territorial) de aglomeração é importante para explicar algumas características do SSI. Essa hipótese é confirmada no estudo de Breschi (2000) ao analisar a relação entre regimes tecnológicos (intensidade tecnológica no setor) e a distribuição espacial da indústria da Itália, França, Reino Unido e Alemanha, entre 1978-1991.

A metodologia do estudo anteriormente citada de Breschi (2000) mostra uma segunda regularidade na análise dos sistemas setoriais: diferenças tecnológicas entre as indústrias. Essa hipótese é identificada nos trabalhos que analisam os fatores envolvidos com a inovação e que variam conforme o setor industrial, o que conduz inevitavelmente a diferenças de aglomeração na indústria. 
As indústrias com maiores taxas de inovação são as mais aglomeradas regionalmente todavia, a necessidade de aglomeração advém de fatores ligados aos custos, como ganhos de escala (KRUGMAN, 1990), captura de transbordamentos de conhecimento (AUDRETSCH, 1998), necessidade de maior volume de P\&D e dependência de P\&D de universidades (AUDRETSCH e FELDMAN, 1996), articulação com capital humano (AUDRETSCH e FELDMAN, 1996), aprendizado para inovar (BOSCHMA, 2005), presença de fornecedores especializados de serviços (FELDMAN e FLORIDA, 1994), cooperação para $\mathrm{P} \& D$ e presença de instituições públicas de pesquisa (FRITSCH e FRANKE, 2004), como laboratórios e centros de pesquisa (FRITSCH e SCHWIRTEN, 1999), redução de custos de transação (MARTIN e OTTAVIANO, 2001). Uma revisão do impacto dos fatores econômicos sobre a aglomeração das empresas, com os citados anteriormente, pode ser vista em Feldman (1999) e Feldman (2000).

A relação entre o progresso tecnológico e aglomeração é direta, sendo apontada como uma tendência de modernização de uma economia por Kuznets (1966). A discussão inconclusiva reside no perfil da aglomeração produtiva e inovativa. Boschma (2005) mostra que as dimensões de proximidade variam, incluindo aspectos de caráter institucional, geográfico, cognitivo, social e organizacional. Estes fatores explicam a necessidade de aglomeração da indústria em função do progresso tecnológico (fator dinâmico) e dos demais fatores estáticos (custos).

Neste sentido, este estudo separa o aspecto geográfico (aproximação territorial da indústria) e analisa o impacto sobre a produtividade total dos fatores das empresas industriais brasileiras, formando a primeira hipótese a ser verificada empiricamente:

Hipótese 1: as diferenças de aglomeração das indústrias brasileiras podem explicar as diferenças de produtividade (e capacidade de inovação) nas

empresas industriais.

Esta hipótese vai ao encontro da primeira regularidade dos Sistemas Setoriais de Inovação. Em relação às diferenças tecnológicas, cada setor reage de maneira diferente em função da necessidade de absorção de competências para inovar. As diferenças de intensidade tecnológica entre os setores explicam a aglomeração e as diferenças de produtividade entre os setores industriais brasileiros. Este problema pode ser resumido na segunda hipótese do artigo.

Hipótese 2: as diferenças de absorção tecnológica variam de acordo com o setor industrial.

A hipótese dois vai ao encontro da do conceito de absorção tecnológica de Cohen e Levinthal (1990). Para os autores a capacidade de absorção da empresa (microeconômica) depende diretamente do ambiente onde a empresa se insere (setor e região, além de outros fatores, como o ambiente institucional, por exemplo, o que foge ao objetivo do artigo).

A terceira regularidade presente nos Sistemas Setoriais de Inovação é o impacto da escala da empresa na capacidade de inovação em função de resposta à escala imposta ao setor e sua intensidade tecnológica, conforme aponta o estudo de Aghion et alli (2002). Os autores definem a hipótese Schumpeteriana de concentração de mercado e inovação como uma curva com formato de U invertido. As empresas maiores inovam mais (até certo ponto), setores mais concentrados, com empresas maiores apresentam maior capacidade de inovação, desde que a competição não seja reduzida em excesso, o que explicaria a redução da inovação em função do menor interesse em competir por parte de empresas em um setor com alto poder de mercado.

A forma de U invertido entre concentração e inovação não é unânime. Archibugi, Evangelista e Simonetti (1995) encontram uma relação positiva entre concentração e tama- 
nho da firma ao analisarem os setores industriais na Itália, ou seja, uma relação linear.

A relação entre concentração de mercado e inovação é expandida para outros fatores econômicos importantes. O estudo de Amable e Verspagen (1995) analisa dados de países da OCDE e verifica diferenças conforme as taxonomias de Pavitt (1984) e Dosi, Pavitt e Soete (1990) na estrutura industrial desses países em função da intensidade tecnológica. Indústrias com maior intensidade tecnológica são mais concentradas em contrapartida, indústrias com menor carga tecnológica são mais competitivas (desconcentradas). Audretsch (1998) mostra que o efeito de transbordamento do conhecimento não é homogêneo entre as empresas, dependendo diretamente do tamanho. Nesse sentido, a maior necessidade de absorção de conhecimento nas indústrias intensivas em tecnologia conduz à aglomeração e concentração de mercado. Esta é a terceira hipótese a ser explorada.

Hipótese 3: as diferenças de concentração dos setores industriais explicam as diferenças de produtividade (e capacidade de inovação) nas empresas industriais. Empiricamente, alguns estudos já avançaram na identificação das diferenças de aglomeração e concentração na indústria brasileira e a sua dinâmica tecnológica.

Bittencourt e Campos (2008) encontram diferenças regionais e setoriais na capacidade de inovação das empresas em função da aglomeração espacial para o estado de Santa Catarina. Feijo et alli (2003) analisam a estrutura industrial nos anos noventa e concluem que a abertura econômica conduziu ao aumento da produtividade. Os setores de alta e média intensidade tecnológica ganharam espaço, o que leva à interpretação de que a dinâmica tecnológica (regime) é proporcional à dinâmica de concentração (e mobilidade espacial). $\mathrm{O}$ aumento da escolaridade e a presença de capital estrangeiro também estão associados à concentração industrial.
Já o estudo de Suzigan et alli (2005) identifica a presença instituições de apoio às empresas para inovar, como cursos superiores (avaliados pelo INEP), cursos tecnológicos, técnicos e de aprendizagem industrial, entidades de classe e o SEBRAE (Serviço Brasileiro de Apoio às Micro e Pequenas Empresas), laboratórios, centros tecnológicos e de P\&D e verificam que estes são concentrados geograficamente em poucos municípios de São Paulo, o que atrai naturalmente as empresas em setores de maior intensidade tecnológica. Dominguez e Ruiz (2006) analisam a indústria brasileira e verificam que quinze aglomerações industriais correspondem a $75 \%$ do valor bruto de transformação industrial brasileira e onze aglomerações tecnológicas correspondem a $78 \%$ das patentes e as duas aglomerações estão ligadas.

Percebe-se um alinhamento entre as três hipóteses identificadas até aqui. A concentração de mercado e a aglomeração espacial são importantes na explicação das diferenças de produtividade (e inovação) entre as empresas.

A próxima seção apresenta o modelo a ser estimado. Com base no estudo de Steingraber (2009) analisa-se o impacto da aglomeração e da concentração (hipóteses 1 e 3) sobre a produtividade total dos fatores das empresas industriais brasileiras no ano de 2005. Para se testar também a hipótese dois levantada anteriormente, utiliza-se um modelo de regressão multinível. Nesse sentido tanto a aglomeração como a concentração interagirão com as competências internas da empresa (competências para inovar e demais características da empresa) para explicar a PTF. Ao se utilizar a regressão multinível, a estimativa por Máxima Verossimilhança é linear, o que restringe a possibilidade de se testar se a relação entre concentração e produtividade também é quadrática (forma de U invertido) assim como na relação entre inovação e concentração de mercado. Portanto, adota-se a visão de Archibugi, Evangelista e Simonetti (1995) que definem a relação como positiva apenas (linear). 


\section{3_0 Modelo}

O modelo de Steingraber $(2009)^{2}$ considera a produtividade total dos fatores da empresa $i$ no setor $j\left(P T F_{i j}\right)$ como função das competências para inovação na empresa $i$ no setor $j\left(C_{i j}\right)$ no ano de 2005:

$$
P T F_{i j}=\propto_{1}+\propto_{2} C_{i j}+e_{i j}
$$

$O$ vetor $C_{i j}$ com as competências para inovação na empresa é composto pelas seguintes variáveis: capital humano inovador, pessoal ocupado com terceiro grau, renda média do trabalhador, tempo médio de escolaridade do trabalhador, número de pedidos de patentes, share, número de empregados e valor das exportações e das importações.

A PTF é calculada pelo método de Resíduo de Abramovitz (1956), conforme defende Antonelli (2003). Dessa forma, a PTF é calculada como a diferença da taxa de mudança do valor do produto, descontado o valor despendido pelos fatores, agora vistos como a taxa de mudança dos gastos com trabalho e o investimento (mudança no estoque de capital da empresa). ${ }^{3}$ A determinação da PTF é dada como:

$$
P T F=d Y-\left(\frac{d y}{d k}\right) d K-\left(\frac{d y}{d l}\right) d L
$$

Onde $d Y$ é a variação do produto (receita líquida de vendas). As derivadas $\left(\frac{d y}{d k}\right)$ e $\left(\frac{d y}{d l}\right)$ são respectivamente as elasticidades do produto em relação ao capital e ao trabalho. Já $d K$ e $d L$ indicam a variação do capital (investimento) e do trabalho. A estimativa da equação (2) foi realizada nos anos de 2004 e 2005, em função da equação se apresentar como uma derivada (taxa de mudança). A vantagem do uso da equação (2) reside na dinâmica de uso de uma taxa de mudança (diferencial) e não de logaritmos, como uma função Cobb-Douglas, além das derivadas parciais não representarem a contribuição marginal do capital e do trabalho no produto, mas a participação relativa destes no produto. A variação do capital, calculada como investimento, facilita a determinação estatística da variável, visto que a PIA não dispõe de uma variável de computação do valor do capital da empresa. A variação de capital (investimento) foi calculada como o valor despendido pela empresa na aquisição de ativos (maquinaria, imóveis, equipamentos e outros ativos imobilizados na PIA) menos as baixas no valor dos mesmos ativos. A variação no trabalho mostra a alteração na estrutura de gastos com a folha de pagamentos (salários, remunerações, benefícios, INSS e outros valores computados na categoria despesa salarial da PIA).

As variáveis que explicam as competências internas da empresa são formadas por meio da base RAIS (renda, escolaridade, tempo de estudo, número de empregados, capital humano inovador, percentual de trabalhadores com terceiro grau de escolaridade, share, experiência do trabalhador na empresa e experiência do trabalhador no setor - estas duas últimas não significativas estatisticamente), da PIA (share, turnover de capital, acesso a insumos internacionais e a própria PTF), SECEX (valor das importações e exportações, em dólares) e INPI (número de pedidos de patentes).

O share da PIA foi calculado em função da participação da receita líquida de vendas da empresa em função do setor. Já o share da RAIS considera a participação do emprego da empresa no setor. Ambas as variáveis foram significativas nas estimativas realizadas todavia, optou-se pelo share da RAIS, visto que as demais variáveis da PIA (insumos internacionais e turnover de capital) não foram significativas estatisticamente na estimativa dos efeitos fixos e foram eliminadas do modelo. 
O vetor com as variáveis de controle setorial (Aglomeração e HHI) pertence à base PIA e identifica a presença de aglomeração produtiva nos setores industriais brasileiros por estado e a concentração nas indústrias com base no índice HHI.

A aglomeração foi calculada como:

$$
A_{j}=\left(\sum_{i=1}^{n} \frac{\text { receita líquida de vendas do setor } j \text { no estado e }}{\text { receita líquida de vendas do setor } j \text { do Brasil }}\right)^{2}
$$

Quanto maior o indicador de aglomeração do setor, maior é a sua concentração em poucos estados. Valores menores indicam uma dispersão do setor em um número maior de estados brasileiros. $\mathrm{O}$ maior valor do indicador é igual a um (1), o que equivale à produção do setor concentrada em um único estado.

O uso da aglomeração por estados e não por meso ou microrregiões (que seriam indicadores mais completos) é justificado em função da necessidade de sigilo dos dados utilizados do IBGE, o uso em regiões menores do que a unidade de estado inviabilizaria a divulgação dos dados (por identificar empresas localizadas em setores com poucas empresas). Neste sentido, o modelo usou a definição de estado como critério de corte regional para os setores.

A distribuição da aglomeração por setores da indústria brasileira é apresentada no gráfico a seguir.

A distribuição da aglomeração produtiva nas indústrias brasileiras, conforme mostra o gráfico 1, é bem distribuída, mas os valores são interpretados como uma estrutura produtiva aglomerada em poucos estados. A maioria das indústrias permaneceu abaixo da mediana (valor 5 no gráfico). Todavia, nenhum setor industrial apresentou valor igual a 0,1 que seria o indicador de uma estrutura pouco concentrada. Os setores industriais com valores inferiores a 0,5 totalizam vinte e oito, com valor de 0,4 são vinte e cinco setores e vinte e quatro setores com valor 0,2 . Acrescentando-se os onze setores com valor de 0,5 , totalizam-se oitenta e oito setores, o que representa $80,73 \%$ dos setores

Gráfico 1_ Distribuição da aglomeração na indústria brasileira no ano de 2005

Aglomeração

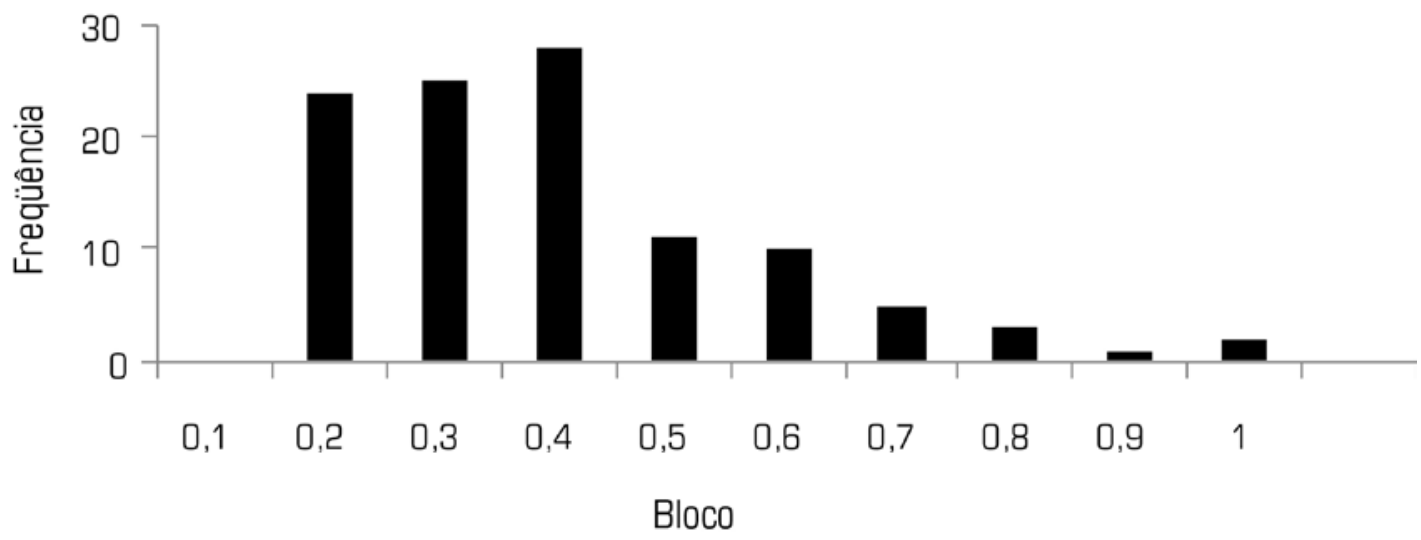

Fonte: Elaboração própria com microdados do IBGE (PIA) 2005. 
industriais brasileiros considerados no modelo (109 no total) e que são aglomerados em poucos estados. Desta forma, os demais 19,27\% dos setores industriais brasileiros (vinte e um setores) são altamente aglomerados em poucos estados do país.

O HHI é calculado para cada setor $j$ da indústria brasileira como:

$$
H H I_{j}=\left(\sum_{i=1}^{n} \frac{\text { receita da empresa } i}{\text { receita do setor } j}\right)^{2}
$$

Quanto maior o valor do HHI, mais concentrada é a indústria. O maior valor possível é igual a um, o que equivale ao setor com apenas uma empresa. A distribuição da variável é apresentada no gráfico a seguir.

O Gráfico 2 mostra que os setores industriais brasileiros não são muito concentrados em relação ao HHI. A maioria dos setores (68) apresentara valor de 0,1, o que representa $62,38 \%$ dos setores industriais brasileiros.
De forma geral, os setores industriais brasileiras apresentam tendência à aglomeração e a concentração, mais para a primeira do que pela segunda, em relação à PTF.

O modelo é estimado pelo método de regressão multinível (ou regressão hierárquica). Para tanto, o segundo nível com o vetor de variáveis de controle setorial Aglomeração e HHI $\left(S_{j}\right)$ no setor $j$ é substituído na equação do primeiro nível. Temos:

$$
\propto_{s}=\beta_{1}+\beta_{2} S_{j}+e_{j}
$$

Onde os parâmetros estimados da primeira equação $\left(\propto_{s}\right)$ são explicados por um termo independente $\left(\beta_{1}\right)$ que é a PTF média da indústria brasileira e a variável setorial $S_{j}$. Os termos independentes no modelo representam a PTF média do nível acima, em função das variáveis serem centralizadas na média. Já éa PTF do setor j e a PTF da empresa $i$ no seu setor é explicada como um desvio desta média

Gráfico 2: Distribuição do HHI na indústria brasileira no ano de 2005

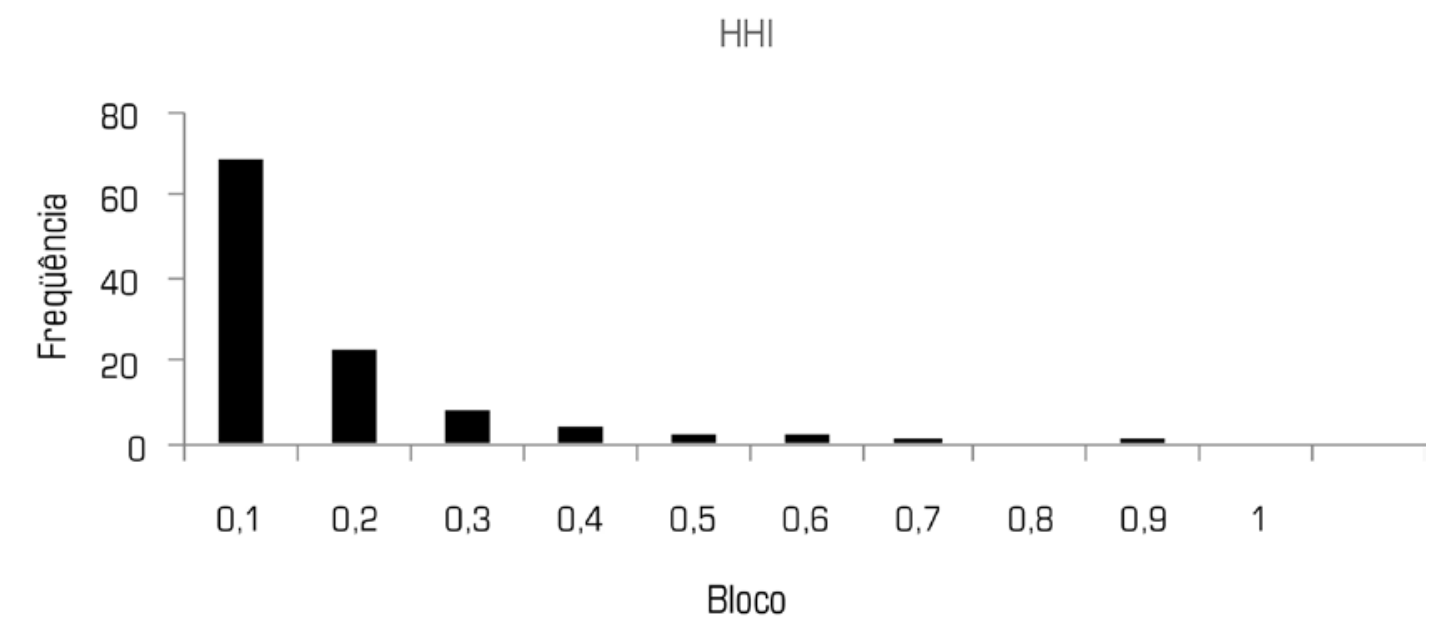

Fonte: Elaboração própria com microdados do IBGE (PIA). 
em função das competências para inovação da referida empresa. Já $\propto_{1}$ é a PTF média da indústria brasileira e a PTF do setor é o desvio desta média em função da aglomeração e do HHI de cada setor. Neste sentido, as variáveis estimadas apresentam desvios positivos (contribuição acima da média na PTF do setor e da indústria respectivamente), ou desvios negativos (contribuição abaixo da média na PTF do setor e da indústria respectivamente).

Substituindo a equação (5) em (1), temos:

$$
P T F_{i j}=\beta_{1}+\beta_{2} S_{j}+e_{j}+\left(\beta_{1}+\beta_{2} S_{j}+e_{j}\right) C_{i j}+e_{i j}(6)
$$

Reagrupando temos:

$$
P T F_{i j}=\beta_{1}+\beta_{1} C_{i j}+\beta_{2} S_{j}+\beta_{2} S_{j} C_{i j}+e_{j} C_{i j}+e_{j}+e_{i j}(7)
$$

Onde a produtividade da empresa $\left(P T F_{i j}\right)$ é explicada pela produtividade do setor $\left(\beta_{1}\right)$, pelos efeitos fixos do setor ( $\beta_{2} S_{j}$ ), os efeitos fixos da empresa $\left(\beta_{1} C_{i j}\right)$, os efeitos fixos na empresa e no setor $\left(\beta_{2} S_{j} C_{i j}\right)$, os efeitos aleatórios $\left(e_{j} C_{i j}\right) \mathrm{e}$ o erro total, composto pela soma do erro na empresa $\left(e_{i j}\right)$ e no setor $\left(e_{j}\right)$.

O modelo multinível é estimado por meio da equação (7).

Além da distribuição, o modelo simples (apenas a PTF em função do termo independente - a PTF nos setores) foi estimado. $\mathrm{O}$ resultado mostra a covariância dos parâmetros, ou seja, quanto do modelo pode ser relacionado à diferença entre empresas e entre setores em relação à produtividade.

Os resultados estimados mostram que a covariância da PTF entre os setores é de 87,E+20 e 62,E+17 para a concentração (HHI) e aglomeração respectivamente, a covariância dos resíduos da PTF foi calculada em 36,E+17 e 4,E+19 para as duas variáveis dependentes. Nesse sentido, os modelos mostram que $96 \%$ da covariância da PTF do modelo de concentração (HHI) é explicada por diferenças entre setores, o que determina que apenas $4 \%$ da covariância da PTF é determinada por diferenças de produtividade entre as empresas do mesmo setor. Para o modelo estimado de aglomeração, a covariância da PTF entre os setores corresponde a $61 \%$ e os demais $39 \%$ são diferenças entre as empresas no mesmo setor.

Estes resultados mostram que as empresas industriais brasileiras diferem em relação às suas PTFs em função do setor. Dentro do mesmo setor, as diferenças são menores. De acordo com o modelo, percebe-se que as diferenças também são relacionadas ao controle setorial. A concentração setorial é mais homogênea em relação à aglomeração espacial das empresas industriais brasileiras, as diferenças de produtividade são mais acentuadas quando são explicadas pelas diferenças de aglomeração e concentração.

Independentemente dos valores, diferenças maiores que $10 \%$ da covariância justificam o uso de modelos de regressão multinível, conforme apontam Gelman e Hill (2006), as diferenças entre setores industriais justificam o uso de agrupamentos conforme a distribuição setorial da CNAE. ${ }^{4}$

Os resultados estimados são apresentados na próxima seção.

\section{4_ Estimativas}

Os valores estimados da equação (4) foram obtidos pelo método de Máxima Verossimilhança (MV), conforme argumenta Hox (2002). O modelo original de Steingraber (2009) seguiu esta metodologia, em função dos estimadores por Mínimos Quadrados Ordinários (MQO) serem inconsistentes na regressão multinível. As estimativas dos efeitos fixos e aleatórios são apresentadas nas Tabelas 1 e 2 a seguir. 
Tabela 1_Resultados estimados dos efeitos fixos na produtividade total dos fatores nas empresas em função da aglomeração e da concentração setorial da indústria brasileira no ano de 2005

\begin{tabular}{|c|c|c|c|c|}
\hline & Aglomeraçãao & & Concentração & \\
\hline Intercepto & $\begin{array}{c}-11200000000 \\
(-2,20)\end{array}$ & 0.0276 & $\begin{array}{c}745048^{N S} \\
(0,64)\end{array}$ & 0.5206 \\
\hline Capital humano inovador & $\begin{array}{c}-1237815 \\
{[-16,66]}\end{array}$ & $<.0001$ & $\begin{array}{c}1667474 \\
(35,92)\end{array}$ & $<.0001$ \\
\hline Pessoal ocupado com terceiro grau & $\begin{array}{l}-582961 \\
(-32,01)\end{array}$ & $<.0001$ & $\begin{array}{r}-367584 \\
(-38,06)\end{array}$ & $<.0001$ \\
\hline Renda média do trabalhador & $\begin{array}{l}13753 \\
(8,31)\end{array}$ & $<.0001$ & $\begin{array}{c}5972.47 \\
(7,78) \\
\end{array}$ & $<.0001$ \\
\hline Número de pedidos de patentes & $\begin{array}{c}-2134681 \\
(-13,23)\end{array}$ & $<.0001$ & $\begin{array}{r}-1719731 \\
(-25,86)\end{array}$ & $<.0001$ \\
\hline Share & $\begin{array}{c}12300000000000 \\
(17,14)\end{array}$ & $<.0001$ & $\begin{array}{c}-415100000000 \\
(-6,36)\end{array}$ & $<.0001$ \\
\hline Número de trabalhadores & $\begin{array}{l}68767 \\
(26,42)\end{array}$ & $<.0001$ & $\begin{array}{l}38212 \\
{[29,76]}\end{array}$ & $<.0001$ \\
\hline Valor exportado & $\begin{array}{l}-0.4053 \\
(-15,49)\end{array}$ & $<.0001$ & $\begin{array}{l}-0.4981 \\
(-23,14) \\
\end{array}$ & $<.0001$ \\
\hline Valor importado & NS & & $\begin{array}{l}0.6257 \\
{[21,41)}\end{array}$ & $<.0001$ \\
\hline Tempo de estudo médio do trabalhador & NS & & $\begin{array}{c}780613 \\
(2,49)\end{array}$ & 0.0127 \\
\hline Aglomeração & $\begin{array}{c}44205144 \\
\text { (2,29) }\end{array}$ & 0.0237 & & \\
\hline $\mathrm{HHI}$ & & & & 0.1770 \\
\hline Capital humano inovador*Aglomeração & $\begin{array}{c}2605090 \\
(21,80)\end{array}$ & $<.0001$ & & \\
\hline $\begin{array}{l}\text { Pessoal ocupado com terceiro } \\
\text { grau*Aglomeração }\end{array}$ & $\begin{array}{c}1760459 \\
(32,88)\end{array}$ & $<.0001$ & & \\
\hline Renda média do trabalhador*Aglomeração & $\begin{array}{l}-31047 \\
{[-6,68]}\end{array}$ & $<.0001$ & & \\
\hline $\begin{array}{l}\text { Número de pedidos de } \\
\text { patentes*Aglomeração }\end{array}$ & $\begin{array}{c}7494760 \\
(15,24)\end{array}$ & $<.0001$ & & \\
\hline Share*Aglomeração & $\begin{array}{c}-3990000000000 \\
(-21,65) \\
\end{array}$ & $<.0001$ & & \\
\hline Número de trabalhadores*Aglomeração & $\begin{array}{r}-140272 \\
(-14,28)\end{array}$ & $<.0001$ & & \\
\hline
\end{tabular}




\begin{tabular}{|c|c|c|c|c|}
\hline \multirow[b]{2}{*}{ Valor exportado*Aglomeração } & \multicolumn{2}{|l|}{ Aglomeraçãao } & \multicolumn{2}{|l|}{ Concentração } \\
\hline & $\begin{array}{c}0.08926 \\
(1,86)\end{array}$ & 0.0636 & & \\
\hline Capital humano inovador* $\mathrm{HHI}$ & & & $\begin{array}{r}-5911374 \\
(-47,65) \\
\end{array}$ & $<.0001$ \\
\hline Pessoal ocupado com terceiro grau*HHI & & & $\begin{array}{c}2335340 \\
(27,30)\end{array}$ & $<.0001$ \\
\hline Renda média do trabalhador*HHI & & & $\begin{array}{l}-89556 \\
(-12,37)\end{array}$ & $<.0001$ \\
\hline Número de pedidos de patentes*HHI & & & $\begin{array}{c}21670490 \\
(36,93)\end{array}$ & $<.0001$ \\
\hline Share*HHI & & & $\begin{array}{c}-1324000000000 \\
(-4,08)\end{array}$ & $<.0001$ \\
\hline Número de trabalhadores*HHI & & & $\begin{array}{r}-33325 \\
(-1,94) \\
\end{array}$ & 0.0529 \\
\hline Valor exportado*HHI & & & $\begin{array}{c}0.3666 \\
(5,05)\end{array}$ & $<.0001$ \\
\hline Valor importado*HHI & & & $\begin{array}{l}0.8132 \\
(13,88)\end{array}$ & $<.0001$ \\
\hline Tempo de estudo médio do trabalhador*HHI & & & $\begin{array}{c}7456046 \\
(1,76)\end{array}$ & 0.0787 \\
\hline -2 Res Log Likelihood & 995003.3 & & 992288.6 & \\
\hline AIC (smaller is better) & 995007.3 & & 992292.6 & \\
\hline AICC (smaller is better) & 995007.3 & & 992292.6 & \\
\hline BIC (smaller is better) & 995012.7 & & 992297.9 & \\
\hline
\end{tabular}

Estatística t entre parênteses.

Fonte: Elaboração própria.

Os dois modelos estimados apresentam semelhanças, mas também algumas divergências específicas da contribuição das variáveis setoriais sobre a produtividade das empresas industriais brasileiras.

Primeiramente, percebe-se que o efeito fixo das variáveis setoriais sobre a produtividade das empresas varia.Para a concentração, o efeito não é significativo estatisticamente, já para a aglomeração a sua contribuição sobre a produti- vidade das empresas é negativa, ou seja, ela contribui menos do que a média da indústria brasileira estimada, fato que indica que apenas a concentração espacial das empresas no seu setor não significa ganho de produtividade em relação aos setores menos concentrados.

Assim como a concentração setorial, os ganhos de produtividade pela concentração espacial são percebidos em relação às competências para inovação na empresa. A con- 
tribuição dos efeitos fixos na empresa mostra estes fatores, na medida em que os parâmetros estimados com estes efeitos fixos são função do controle setorial.

A renda do trabalhador e o número de empregados apresentam ganhos de produtividade acima da média da indústria brasileira em relação às empresas.

A participação de trabalhadores com $3^{\circ}$ grau na empresa, o número de pedidos de patentes e as exportações apresentam ganhos de produtividade abaixo da média da indústria brasileira em relação às empresas.

As diferenças surgem no capital humano inovador, com ganhos acima da média quando o controle setorial foi introduzido e abaixo da média para a concentração espacial. $\mathrm{O}$ share apresentou resultados opostos respectivamente (acima para a aglomeração e abaixo para a concentração). As importações e o tempo de estudo do trabalhador não foram significativos para o controle setorial no modelo de aglomeração e foram positivos (cima da média) para o modelo de concentração na produtividade das empresas.

Estas diferenças mostram que a produtividade das empresas reage do modo diferente conforme o controle setorial observado sobre as competências das empresas. Algumas competências geram maior impacto sobre a produtividade da empresa, enquanto outras geram impactos menores (abaixo da média).

Os efeitos fixos do setor sobre as competências da empresa são praticamente opostos aos efeitos fixos da empresa sobre a sua produtividade. Enquanto na empresa a renda do trabalhador e o número de empregados determinavam ganhos de produtividade acima da média, no setor estes ganhos são abaixo da média para as duas variáveis de controle setorial. As exportações, o número de pedidos de patentes e o percentual de trabalhadores com terceiro grau apresentaram ganhos de produtividade acima da média no setor (na empresa estes ganhos estavam abaixo da média).
O capital humano inovador apresentou sinais invertidos para cada modelo estimado em relação aos efeitos fixos da empresa e do setor, já o share foi estimado com impactos abaixo da média para o controle setorial. Na empresa apenas o modelo de concentração setorial apresentou ganhos de produtividade abaixo da média para esta competência da empresa para inovar.

A importação e o tempo de estudo do trabalhador apresentaram ganhos de produtividade acima da média no setor e na empresa, para o controle setorial de concentração setorial.

Percebe-se a diferença entre o impacto da empresa e do setor sobre as competências para inovar na produtividade. Esta diferença mostra que o setor apresenta comportamento próprio, neste caso, a aglomeração espacial e a concentração setorial das empresas definem diferentes resultados sobre o desempenho das empresas.

A estimativa desenvolvida comprova que as políticas públicas de inovação devem levar em consideração a aglomeração espacial e setorial das empresas. Neste sentido, os efeitos aleatórios estimados permitem identificar os setores mais sensíveis as duas variáveis de controle setorial. Os resultados estimados significativos estatisticamente são apresentados na Tabela 2.

Trinta e dois setores apresentaram diferenças significativas estatisticamente de ganhos de produtividade (acima e abaixo) da média da indústria brasileira em relação à aglomeração regional e à concentração setorial. Nestes, vinte e oito setores foram sensíveis à aglomeração e vinte e três à concentração.

Os setores não significativos estatisticamente estão dentro da produtividade média da indústria brasileira.

Entre os setores significativos estatisticamente existem setores sensíveis à escala e de baixa intensidade tecnológica, como a extração de carvão mineral (100), assim como setores menos sensíveis a fatores estáticos de produtividade $\mathrm{e}$ 
Tabela 2_Resultados dos efeitos aleatórios da aglomeração e

da concentração setorial sobre a PTF das empresas industriais brasileiras no ano de 2005

\begin{tabular}{|c|c|c|c|c|}
\hline Setor CNAE & Aglomeração & & Concentração & (Cor \\
\hline 100 & $\begin{array}{c}1190000000000 \\
(3,81)\end{array}$ & $<.0001$ & $\begin{array}{c}2420000000000 \\
(1,73)\end{array}$ & 0.0836 \\
\hline 112 & $\begin{array}{c}50702314 \\
(2,33)\end{array}$ & 0.0200 & NS & \\
\hline 131 & $\begin{array}{c}97420370 \\
(4,97)\end{array}$ & $<.0001$ & $\begin{array}{c}2990000000000 \\
(6,57)\end{array}$ & $<.0001$ \\
\hline 132 & NS & & $\begin{array}{c}1590000000000 \\
(2,42)\end{array}$ & 0.0155 \\
\hline 151 & $\begin{array}{c}51188098 \\
(2,58)\end{array}$ & 0.0098 & $\begin{array}{c}1640000000000 \\
(2,48)\end{array}$ & 0.0132 \\
\hline 152 & $\begin{array}{c}-51400000000 \\
(-2,64)\end{array}$ & 0.0084 & $\begin{array}{c}-165000000000 \\
(-2,77)\end{array}$ & 0.0057 \\
\hline 153 & $\begin{array}{c}-244000000000 \\
(-9,68)\end{array}$ & $<.0001$ & $\begin{array}{r}-5.33 \mathrm{E} 8 \\
(-9,56) \\
\end{array}$ & $<.0001$ \\
\hline 154 & $\begin{array}{c}44125847 \\
(2,41)\end{array}$ & 0.0162 & NS & \\
\hline 156 & $\begin{array}{c}-87000000000 \\
(-3,14)\end{array}$ & 0.0017 & $\begin{array}{c}-468000000000 \\
(-2,05)\end{array}$ & 0.0402 \\
\hline 159 & NS & & $\begin{array}{c}-126000000000 \\
(-2,41)\end{array}$ & 0.0161 \\
\hline 174 & $\begin{array}{c}-41700000000 \\
(-1,69)\end{array}$ & 0.0917 & $\begin{array}{c}-110000000000 \\
{[-1,91)}\end{array}$ & 0.0561 \\
\hline 221 & NS & & $\begin{array}{c}2610000000000 \\
(2,17)\end{array}$ & 0.0303 \\
\hline 223 & $\begin{array}{c}86423684 \\
(3,41)\end{array}$ & 0.0006 & $\begin{array}{c}1660000000000 \\
(2,44)\end{array}$ & 0.0146 \\
\hline 241 & $\begin{array}{c}-42400000000 \\
(-2,00)\end{array}$ & 0.0456 & $\begin{array}{c}-443000000000 \\
(-5,43)\end{array}$ & $<.0001$ \\
\hline 242 & $\begin{array}{c}37037974 \\
(2,07)\end{array}$ & 0.0384 & NS & \\
\hline 245 & $\begin{array}{c}-23700000000 \\
(-1,81)\end{array}$ & 0.0708 & $\begin{array}{c}4870000000000 \\
(3,24)\end{array}$ & 0.0012 \\
\hline 246 & $\begin{array}{c}-257000000000 \\
(-12,02)\end{array}$ & $<.0001$ & $\begin{array}{c}-1020000000000 \\
(-11,94)\end{array}$ & $<.0001$ \\
\hline 262 & $\begin{array}{c}-208000000000 \\
(-4,03)\end{array}$ & $<.0001$ & $\begin{array}{c}-602000000000 \\
(-4,65)\end{array}$ & $<.0001$ \\
\hline 272 & $\begin{array}{c}37320548 \\
(1,86)\end{array}$ & 0.0627 & $\begin{array}{r}-1.66 \mathrm{E} 8 \\
(-2,16)\end{array}$ & 0.0309 \\
\hline
\end{tabular}




\begin{tabular}{|c|c|c|c|c|}
\hline Setor CNAE & Aglomeração & & Concentração & (Con \\
\hline 273 & $\begin{array}{c}42189499 \\
(1,65)\end{array}$ & 0.0997 & $\begin{array}{c}1170000000000 \\
(1,75)\end{array}$ & 0.0807 \\
\hline 274 & NS & & $\begin{array}{r}-1.52 \mathrm{E} 8 \\
(-1,74) \\
\end{array}$ & 0.0826 \\
\hline 293 & $\begin{array}{c}46403224 \\
(-4,26)\end{array}$ & $<.0001$ & $\begin{array}{c}-208000000000 \\
(-4,07)\end{array}$ & $<.0001$ \\
\hline 298 & $\begin{array}{c}63590355 \\
(1,76)\end{array}$ & 0.0776 & $\begin{array}{c}-163000000000 \\
(-2,94)\end{array}$ & 0.0032 \\
\hline 311 & $\begin{array}{c}63590355 \\
(2,36)\end{array}$ & 0.0184 & NS & \\
\hline 312 & $\begin{array}{c}-27300000000 \\
(-1,83)\end{array}$ & 0.0675 & NS & \\
\hline 322 & $\begin{array}{c}1250000000000 \\
(6,24)\end{array}$ & $<.0001$ & NS & \\
\hline 329 & $\begin{array}{c}-297000000000 \\
(-7,92)\end{array}$ & $<.0001$ & $\begin{array}{c}-267000000000 \\
(-3,51)\end{array}$ & 0.0005 \\
\hline 335 & $\begin{array}{c}74066404 \\
(2,26)\end{array}$ & 0.0240 & NS & \\
\hline 341 & $\begin{array}{c}4660000000000 \\
\text { (17,32) }\end{array}$ & $<.0001$ & $\begin{array}{c}21700000000000 \\
(23,46)\end{array}$ & $<.0001$ \\
\hline 342 & $\begin{array}{c}57050152 \\
(1,96)\end{array}$ & 0.0495 & $\begin{array}{c}3330000000000 \\
(4,33)\end{array}$ & $<.0001$ \\
\hline 352 & $\begin{array}{c}40861983 \\
(1,88)\end{array}$ & 0.0597 & NS & \\
\hline 353 & $\begin{array}{c}-120000000000 \\
(-5,38)\end{array}$ & $<.0001$ & NS & \\
\hline
\end{tabular}

Estatística t entre parênteses.

Fonte: Elaboração própria.

mais dinâmicos tecnologicamente na indústria brasileira, como a fabricação de automóveis.

Neste sentido, podemos afirmar que a tecnologia e o conhecimento não são os principais fatores de dinamismo espacial e de concentração de mercado.

Analisando individualmente os setores, percebemos três grupos distintos: a) setores com ganhos de produtividade acima da média para a aglomeração e a concentração; b) setores com ganhos de produtividade abaixo da média para a aglomeração e a concentração; c) setores com ganhos de produtividade acima da média para a aglomeração e abaixo da média para a concentração. Apenas a fabricação de produtos farmacêuticos (245) apresentou ganhos de produtividade abaixo da média para a aglomeração e acima da média para a concentração. Este resultado mostra que a indústria farmacêutica brasileira é importadora de tecnologia, na medida em que é dominada por empresas multinacionais que não formam capital social com o território, 
as universidades e outras instituições, dependendo mais do seu poder de mercado.

Os setores com ganhos de produtividade acima da média na aglomeração e a concentração são: extração de carvão mineral (100), extração de minério de ferro (131), abate e preparação de produtos de carne e de pescado (151), reprodução de material gravado (223), fabricação de tubos (273), fabricação de automóveis (341), fabricação de caminhões e ônibus (342). Igualmente a extração de minerais metálicos não ferrosos (132) e a edição e impressão (221) apresentaram ganhos de produtividade apenas em relação à concentração. Já a fabricação de laticínios (154), fabricação de produtos químicos orgânicos (242), fabricação de geradores, transformadores e motores elétricos (311), fabricação de aparelhos e equipamentos de telefonia e radiotelefonia e de transmissões de televisão e rádio (322), fabricação de cronômetros e relógios (335) e a construção, montagem e reparação de veículos ferroviários (352) apresentaram ganhos de produtividade apenas para a aglomeração.

Estas indústrias são sensíveis aos ganhos de produtividade de tamanho e aglomeração espacial. Desta forma, não somente a escala é um fator importante para ganhos de produtividade e capacidade de inovação, como também o desenvolvimento da cadeia de fornecedores, o que explica mais os ganhos de produtividade espaciais em função de integração vertical do que pela presença de instituições de conhecimento.

Os setores com ganhos de produtividade abaixo da média da indústria brasileira são: processamento, preservação e produção de conservas, frutas, legumes e verduras (152), produção de óleos, gorduras vegetais e animais (153), fabricação e refino de açúcar (156), fabricação de artefatos têxteis, incluindo tecelagem (174), fabricação de produtos químicos inorgânicos (241), fabricação de defensivos agrícolas (246), fabricação de cimento (262), manutenção e reparação de aparelhos e equipamentos de telefonia e radiotelefonia e de transmissão de televisão e rádio - exceto telefones (329). Os setores de fabricação de bebidas (159) e a metalurgia de metais não ferrosos (274) apresentaram ganhos de produtividade abaixo da média apenas para a concentração e a fabricação de material eletrônico básico (321) e a construção, montagem e reparação de aeronaves (353) apresentaram ganhos de produtividade abaixo da média para a aglomeração.

Estas últimas indústrias apresentam dispersão regional em diversas áreas do agronegócio e do setor aeroespacial. Percebemos que a simples aglomeração espacial não gera ganhos de produtividade nestes setores. Não sugerimos a adoção de políticas de concentração para tais setores, mas a adoção de políticas públicas de desenvolvimento que considerem a fragilidade estrutural por conta da sua dispersão regional, favorecendo a formação de capital social em Arranjos Produtivos e Inovativos Locais que fortalecem a cadeia produtiva fragmentada e o tamanho reduzido da maioria das empresas nestes setores.

Por fim, os setores com ganhos de produtividade acima da média para a aglomeração e abaixo da média para a concentração são: siderurgia (272), fabricação de tratores e de máquinas e equipamentos para a agricultura, avicultura e obtenção de produtos animais (293) e fabricação de eletrodomésticos (298). Estes setores se mostram competitivos em função da concentração regional e da presença de capital humano qualificado. Novamente, o desenvolvimento de políticas públicas de inovação, formação de capital social e humano para estes setores deve respeitar as suas especificidades regionais.

Claramente, não se encontrou semelhança entre os setores intensivos em tecnologia apontados nos estudos de Pavitt (1984) e Dosi et alli (1990) em relação aos ganhos de produtividade das empresas industriais brasileiras em fun- 
ção da aglomeração e da concentração setorial. Certamente, fatores estruturais estáticos, como custos, conduzem à concentração e à aglomeração e não fatores dinâmicos, como a necessidade de absorção de conhecimento. A presença de setores intensivos em escala, como a siderurgia, com ganhos de produtividade acima da média e não o setor aeronáutico (ganhos de produtividade abaixo da média nacional), nos diz que os ganhos de competitividade na indústria brasileira não são originados apenas pelas competências internas dedicadas à inovação.

Este resultado abre espaço para o atual debate sobre Política Industrial e Tecnológica no Brasil. Primeiramente porque reforça a presença de fatores estruturais que não são homogêneos, como tamanho das empresas e sua concentração de mercado e a aglomeração espacial como determinantes nos ganhos de produtividade e o desenvolvimento de inovações. Desta forma, tais fatores devem ser levados em consideração na determinação dos incentivos e políticas públicas para inovação e competitividade industrial. Segundo, grande parte dos setores industriais com ganhos de produtividade acima da média da indústria brasileira é formado por setores de baixa intensidade tecnológica e não são estratégicos para a definição de políticas públicas de inovação, como o setor de carnes (indústria alimentícia), por exemplo.

Identifica-se a necessidade de estudos específicos para a definição de estratégias de inovação e inserção internacional de tais setores, como os ganhos de produtividade facilitam a capacidade de inovação da indústria e estratégias específicas para tais setores podem resultar em ganhos mais acentuados de exportação.

Causa preocupação ainda a falta de políticas industriais (e tecnológicas) claras para os setores com menor capacidade de aglomeração e concentração. Reflexo do descaso brasileiro com uma Política Industrial para a Pequena e Média
Empresa. Dispersas e com menor escala, tais empresas não acessam com facilidade os recursos disponíveis para inovação no Brasil. Conclui-se que poucos setores se destacaram individualmente no exercício empírico realizado, os que se destacam não são os mais dinâmicos tecnologicamente. Uma nova abordagem de estratégia de inovação na indústria brasileira que contemple as perspectivas setorial e territorial deve ser urgentemente debatida na academia, governo e setor empresarial.

\section{5_Conclusão}

Este artigo analisou o impacto da aglomeração e da concentração setorial sobre a produtividade total dos fatores das empresas industriais brasileiras, por meio das suas competências para inovar no ano de 2005.

A literatura sobre inovação debate o papel do território e do tamanho das empresas sobre a produtividade e a capacidade de inovação. A aglomeração espacial implica ganhos de produtividade em função de externalidades positivas, economias de escala e desenvolvimento de uma rede de competências para inovar, como a presença de universidades e outros atores importantes na cadeia produtiva e na geração de conhecimento. A influência da concentração de mercado é analisada por meio do HHI e mostra como o tamanho relativo da empresa no seu setor apresenta vantagens no desenvolvimento de competência para inovar e impacta positivamente a produtividade da empresa.

O modelo estimado confirmou a influência do setor sobre as competências para inovação na empresa e na sua produtividade.

Primeiramente, percebe-se que as competências para inovar na empresa impactam positivamente o aumento de produtividade nas empresas industriais. Todavia, algumas competências, como as exportações, o número de pedidos 
de patentes e o percentual de trabalhadores com terceiro grau apresentam ganhos de produtividade abaixo da média da indústria brasileira.

Todavia, com o controle setorial de aglomeração e concentração os sinais praticamente ficam invertidos, o que mostra que as competências para inovação que geram ganhos de produtividade na empresa não geram estes ganhos no setor e vice-versa, as competências para inovar importantes setorialmente não geram os mesmos ganhos de produtividade na empresa.

Este resultado reforça a importância de que a inovação apresenta fatores específicos e que não podem ser generalizados, desta forma, a definição de políticas públicas para inovação e produtividade deve interagir com estas diferenças, estabelecendo incentivos para os fatores na esfera micro e mesoeconômica.

O modelo analisado não considera a esfera macroeconômica. Esta poderia ser analisada futuramente dentro do modelo por meio do terceiro nível na regressão multinível. Certamente, algumas competências afetam mais a produtividade das empresas no cenário macroeconômico.

Os resultados estimados também mostram que as empresas industriais brasileiras dependem de fatores setoriais para o incremento de sua produtividade, todavia, tais fatores são atualmente desconsiderados na agenda de política pública para o desenvolvimento econômico.

Primeiramente, se o HHI mostra que alguns setores dependem da concentração setorial para alavancar a produtividade e inovação, a política de regulação (que ainda caminha a passos lentos no Brasil) deve ser debatida e avançar a passos largos para garantir a promoção de inovações e ganhos de produtividade em setores concentrados setorialmente e com grandes empresas. $\mathrm{O}$ atual cenário de regulação econômica e baixo incentivo à internacionalização das grandes empresas industriais brasileiras mostra como a falta de políticas públicas diretas para setores e (grandes) empresas concentradas restringe a competitividade brasileira, além de ser um assunto com pouca tradição no debate econômico e político brasileiro. Por outro lado, os setores com desempenho de produtividade igual à média brasileira foram maioria conforme os resultados estimados apontam. Tal resultado conduz a uma reflexão inevitável: percebe-se que a grande empresa possui vantagem para inovar, os setores mais concentrados encontram essa mesma facilidade, todavia, como ficam as pequenas e médias empresas, bem como os setores menos concentrados economicamente? A agenda de Política Industrial e Tecnológica brasileira precisa debater urgentemente uma estratégia de inovação e produtividade específica para tais setores.

Da mesma maneira, se a aglomeração espacial explica o desempenho da produtividade e das competências para inovação em muitos setores, a política de desconcentração industrial brasileira, promovida pela Guerra Fiscal entre estados e a mudança de muitas empresas por incentivos fiscais diminuem a competitividade nestes setores. A nossa recomendação é da adoção de políticas públicas de formação de capital social e humano que promovam as competências para inovação nos setores dependentes da aglomeração, para que a aproximação territorial não seja a única explicação para o desenvolvimento de competências dinâmicas essenciais, incrementando a formação de avanços tecnológicos.

Os resultados deste estudo destacam o importante papel das exportações, do percentual de trabalhadores com terceiro grau e o número de pedidos de patentes como competências para inovação nas empresas que dependem da aglomeração e da concentração setoriais na indústria brasileira. Estas variáveis merecem estudos próprios unindo as esferas micro, meso e macroeconômica e seus impactos na produtividade e capacidade de inovação de forma sistêmica. 
Por fim, percebemos que a adoção de políticas públicas para a inovação no Brasil, definidas por critérios microeconômicos não é eficiente. $\mathrm{O}$ país está subestimando as características regionais e setoriais particulares que explicam grande parte do desempenho competitivo na atual economia baseada no conhecimento e menos em fatores estáticos. Mais estudos devem aprofundar como se dá a relação entre as características setoriais e as competências para inovação na empresa, formando a base competitiva da indústria nacional, bem como definir instrumentos e incentivos que ressaltem a capacidade de inovação e da produtividade neste cenário.

\section{Notas}

${ }^{1} \mathrm{O}$ modelo considerado foi construído com a elaboração de um projeto de pesquisa do IPEA com o uso de microdados do IBGE. A posse dos dados utilizados não é dos autores. Agradecemos ao Professor Doutor Mário Sérgio Salerno (Observatório da Inovação e Competitividade) e ao Doutor João Alberto de Negri (IPEA) pelo apoio ao projeto.

${ }^{2}$ Os resultados sobre as diferenças setoriais de produtividade e inovação estão no estudo de Steingraber e Gonçalves (2010).

\author{
${ }^{3}$ Importante destacar que o \\ resíduo não é de Solow (Solow, \\ 1956 e Solow, 1957). Neste sentido, a \\ interpretação da PTF é a mesma, \\ todavia, as demais variáveis \\ derivadas não são interpretadas \\ como a participação marginal no \\ produto, mas como a participação \\ relativa no produto. \\ ${ }^{4}$ Uma regressão hierarquica capta \\ endogenamente a influência \\ de agrupamentos da variável \\ considerada. Neste sentido, o \\ cálculo de uma produtividade \\ média não está errado, mas des \\ considera as diferenças existentes \\ entre os setores industriais.
}


ABRAMOVITZ, M. Resource and output trends in the United States since 1870. American Economic Review, v. 46, p. 5-23, 1956.

AGHION, P; BLOOM, N; BLUNDELL, R; GRIFFITH, R; HOWITT, P. Competition and innovation: an inverted $\mathrm{U}$ relationship. The Institute for Fiscal Studies, working paper WP02/04, 2002.

AMABLE, B; VERSPAGEN, B. The role of technology in market shares dynamics. Applied Economics, v. 27, p. 197-204, 1995

ANTONELLI, C. The economics of innovation, new technologies and structural change. London: Routledge, 2003.

ARCHIBUGI, D; EVANGELISTA, R; SIMONETTI, R. Concentration, firm size and innovation: evidence from innovation costs. Technovation, volume 15, number 3 , p. 153-163, 1995.

\section{AUDRETSCH, D.B.}

Agglomeration and the location of innovative activity. Oxford Review of Economic Policy, volume 14, number 2, p. 18-29, 1998.

AUDRETSCH, D.B; FELDMAN, M.P. R\&D spillovers and the geography of innovation and production. The American Economic Review, volume 86, number 3, p. 630-640, 1996.
BITTENCOURT, P.F; CAMPOS, R.R. Processos de aprendizagem em empresas inovadoras em aglomerações produtivas: uma análise exploratória dos dados da Pintec para Santa

Catarina. Nova Economia, v. 18, n. 3, p. 471-500, 2008 .

BOSCHMA, R. A. Proximity and innovation: a critical assessment. Regional Studies, volume 39 , number 1 , p. 61-74, 2005.

BRESCHI, S. The geography of innovation: a cross-sector analysis. Regional Studies, volume 34, number 3, p. 231-229, 2000.

BRESCHI, S; MALERBA, F.

Sectoral innovation systems: technological regimes, Schumpeterian dynamics, and spatial boundaries. In: EDQUIST, C. Systems of innovation: technologies, institutions, and organizations. New York: Routledge, p. 130-156, 1997.

CARLSSON, B; JACOBSSON, S; HOLMÉN, M; RICKNE, A Innovation systems: analytical and methodological issues. Research Policy, volume 31, p. $233-245,2002$.
COHEN, W.M.; LEVINTHAL, D.A. Absorptive capacity: a new perspective on learning and innovation. Administrative Science Quarterly, volume 35, number 1, Special Issue: Technology, Organizations, and Innovations, p. 128-152, 1990.

DOMINGUEZ, E.P; RUIZ, RM. Aglomerações industriais e tecnológicas: origem do capital, inovação e localização. Economia e Sociedade, volume 15, número 3, p. 515-543, 2006.

DOSI, G; PAVITT, K; SOETE, L. The economics of technical change and international trade. London: Harvester Wheatshead, 1990.

FEIJO, C.A; CARVALHO, P.G.M de; RODRIGUEZ, M.S. Concentração industrial e produtividade do trabalho na indústria de transformação nos anos 90: evidências empíricas. Econômica, v. 4, n. 1, p. 19-52, 2003.

FELDMAN, M.P. The new economics of innovation, spillovers and agglomeration: a review of empirical studies. Economic Innovation and New Technology, volume 8, p. 5-25, 1999.
FELDMAN, M.P. Location and innovation: the new economic geography on innovation, spillovers, and agglomeration. In: CLARK, G. L.; FELDMAN, M. P.; GERTLER, M. S. The Oxford handbook of economic geography. Oxford: Oxford University, p. 373-394, 2000.

FELDMAN, M.P; FLORIDA, $R$. The geography sources of innovation: technological infrastructure and product innovation in the United States. Annals of the Association of American Geographers, volume 84, number 2, p. 210-229, 1994.

FRITSCH, M; FRANKE, G. Innovation, regional knowledge spillovers and R\&D cooperation. Research Policy, volume 33,

p. 245-255, 2004.

FRITSCH, M; SCHWIRTEN, C. Enterprise-university cooperation and the role of public research institutions on regional innovation systems. Industry and Innovation, volume 6 , number 1 ,

p. $69-83,1999$.

GELMAN, A; HILL, J. Data analysis using regression and multilevel/ hierarchical models. Cambridge: Cambridge University, 2006.

HOX, J. Multilevel analysis techniques and applications. New Jersey: Lawrence Erlbaum Associates, 2002. 


\section{KUZNETS, S. Modern}

economic growth: rate, structure and spread. New Heaven:

Yale University, 1966.

KRUGMAN, P. Increasing returns and economic geography. Journal of Political Economy, volume 99, number 3,

p. 483-499, 1990.

MALERBA, F. Sectoral systems of innovation and production. Research Policy, volume 31, p. 247-264, 2002.

MARTIN, P.; OTTAVIANO, G. I. P. Growth and agglomeration. International Economic Review, volume 42, number 4, p. 947-968, 2001.

PAVITT, K. Sectoral patterns of technical change: towards a taxonomy and a theory. Research Policy, volume 13, p. 343-373, 1984.

SOLOW, R.M. A contribution to the theory of economic growth. The Quarterly Journal of Economics, volume 70, number 1, p. 65-94, 1956.
SOLOW, R.M. Technical change and the aggregate production function. The Review of Economics and Statistics, volume 39, number 3 , p. 312-320, 1957.

STEINGRABER, R. Inovação e produtividade: o papel dos sistemas de inovação para a indústria brasileira.

Curitiba: UFPR - Tese de

Doutorado, 2009.

STEINGRABER, R; GONÇALVES $\mathrm{F}$ de O. Inovação, instituições e capital social na produtividade total dos fatores na indústria brasileira em 2005. In: SALERNO, M.S; NEGRI, JA de; TURCHI,

L.M; MORAIS, LM de. Inovação: estudos de jovens pesquisadores brasileiros (volume 2). São Paulo: Editora Papagaio, p. 119-147, 2010.

SUZIGAN, W; CERRÓN, A.P.M; DIEGUES JÚNIOR, A.C. Localização, inovação e aglomeração: o papel das instituições de apoio às empresas no estado de São Paulo. São Paulo em Perspectiva, v. 19, n. 2, p. 86-100, 2005.
E-mail de contato dos autores:

ronivaldo.ufsc@gmail.com

flaviogonsalves@hotmail.com

Artigo recebido em janeiro de 2011 aprovado em dezembro de 2012. 